\title{
Identification of Known and Novel PTCH Mutations in Both Syndromic and Non-syndromic Keratocystic Odontogenic Tumors
}

\author{
Shuang Pan, Li-li Xu, Li-sha Sun, Tie-jun Li* \\ Department of Oral Pathology, Peking University School and Hospital of Stomatology, Beijing, China
}

\begin{abstract}
Shuang Pan, Li-li Xu, Li-sha Sun, Tie-jun Li. Identification of Known and Novel PTCH Mutations in Both Syndromic and Non-syndromic Keratocystic Odontogenic Tumors. International Journal of Oral Science, 1(1): 34-38, 2009
\end{abstract}

Aim To clarify the role of PTCH in patients with NBCCSrelated and non-sydromic keratocystic odontogenic tumors. Methodology Mutation analysis was undertaken in 8 sporadic and 4 NBCCS-associated KCOTs.

Results Four novel and two known mutations were identi- fied in 2 sporadic and 3 syndromic cases, two of which being germline mutations (c.2179delT, c.2824delC) and 4 somatic mutations (c.3162dupG, c.1362-1374dup, c.1012 C $>$ T, c.403C $>$ T).

Conclusion Our findings suggest that defects of $P T C H$ are associated with the pathogenesis of syndromic as well as a subset of non-syndromic KCOTs.

Keywords keratocystic odontogenic tumor, mutation, nevoid basal cell carcinoma syndrome, $P T C H$

Document code: A CLC number: Q754 Received Nov.21,2008; Revision accepted Jan.6,2009

\section{Introduction}

Nevoid basal cell carcinoma syndrome (NBC$\mathrm{CS}$, also known as Gorlin syndrome, OMIM NO. 109400) is a rare autosomal dominant disorder characterized by multiple basal cell carcinomas, keratocystic odontogenic tumors (KCOTs), medulloblastoma, ovarian fibroma, and developmental defects such as palmar/plantar pits, calcified falx cerebri, bridged sella, bifid ribs, scoliosis, and frontal bossing, etc (Gorlin, 2004). PTCH gene (OMIM NO.601309), the human homologue of the Drosophila segment polarity gene, has been identified as the gene responsible for NBCCS. PTCH gene consists of 23 exons spanning approximately $34 \mathrm{kbp}$. It encodes a 1447 amino acid protein containing 12 transmembrane-spanning domains and 2 large extracellular loops as well as a large intracellular loop (Calzada-Wack et al., 2002). It is involved in the Sonic hedgehog (SHH) signaling pathway which has been implicated in the formation of embryonic structures and tumorigenesis. Mutation of $P T C H$ resulting in aberrant activity of
SHH pathway may lead to NBCCS as well as some related sporadic tumors, such as basal cell carcinoma, KCOTs, medulloblastoma, and trichoepithelioma et al (Bale et al., 2001; Pietsch et al., 1997; Wolter et al., 1997).

KCOTs are common aggressive cystic jaw tumors with a high growth potential and a propensity for recurrence. The majority of KCOTs arise sporadically in single form while multiple lesions often occur when associated with NBCCS. It has been reported that nearly $75 \%$ NBCCS-associated KCOTs harbored $P T C H$ mutations, while only a few reports documented $P T C H$ mutations in Chinese patients with sporadic KCOTs (Gu et al., 2006; Sun et al., 2008). The aim of this study was to search for $P T C H$ mutations in both nonsyndromic and syndrome-associated KCOTs. Mutations in 2 out of 8 sporadic KCOTs and 3 out of 4 NBCCS-associated KCOTs suggested that defects of $P T C H$ may play a crucial role in the genesis of both sporadic and NBCCS-associated KCOTs. 


\section{Methods and materials}

\section{Patients and samples}

Fragments from KCOT samples were obtained from Peking University Hospital and School of Stomatology. Eight lesions were histological classified as sporadic $\mathrm{KCOT}(\mathrm{KC} 1-\mathrm{KC} 8)$, and 4 were from patients with NBCCS (NB1-NB4) diagnosed according to the established criteria (Kimonis et al., 1997). Fresh tissue specimens as well as their corresponding peripheral blood samples were collected for subsequent analysis. The study protocol was approved by the Ethical Committee of Peking University Health Science Center. Informed consents were obtained from all patients.

\section{DNA extraction and polymerase chain reaction}

Genomic DNA from tumors and peripheral blood was extracted with a QIAamp DNA Mini Kit (Qiagen, Germany). All 23 exons and the exon-intron boundaries of the PTCH gene were amplified by polymerase chain reaction (PCR) with specific primers described previously ( $\mathrm{Gu}$ et al., 2006; Sun et al., 2008). PCR was performed in a volume of $50 \mu \mathrm{L}$ containing $100 \mathrm{ng}$ of DNA, $10 \mathrm{pmol} / \mathrm{L}$ of each primer, $2.5 \mathrm{mmol} / \mathrm{L}$ of dNTPs, 1 unit of Taq DNA polymerase in PCR buffer. Amplification was performed for 35 cycles at $95^{\circ} \mathrm{C}$ for 30 seconds, $61^{\circ} \mathrm{C}$ for 30 seconds and $72^{\circ} \mathrm{C}$ for 45 seconds to 3 minutes.

\section{Denaturing highperformance liquid chromato- gramphy analysis and sequencing}

PCR products were separated through $2 \%$ agarose gels and purified using the Takara gel extraction kit. PCR products were analyzed by denaturing highperformance liquid chromatography (DHPLC) to screen for PTCH mutations. Samples showing an abnormal elution profile were subjected to direct sequencing. When insertion or deletion of multiple nucleotides were detected by direct sequencing, the purified products were ligated into pGEM-T vector (Promega, USA), and the resulting clones were sequenced on an ABI PRISM 3100 Genetic Analyzer (Applied Biosystems, USA).
Total RNA extraction and reverse transcriptase polymerase chain reaction

Where two different heterozygous mutations were detected in one case, reverse transcriptase polymerase chain reaction (RT-PCR) was used to determine whether two variants were located in one or two alleles. Total RNA was extraction from tumor of NB3 patient by means of Trizol ${ }^{\mathrm{TM}}$ reagent (Invitrogen, USA). cDNA were synthesized with Superscript $^{\mathrm{TM}}$ Ш First-Strand synthesis system (Invitrogen, USA). A $2 \mu \mathrm{L}$ quantity cDNA was used to amplify segment (E3-17) designed to cover the region carrying the two mutational changes by forward primer 5'-TGGGATTAAAAGCAGCGAA C-3' and reverse primer 5'-AGCCCCAGGCTCGT ATAGTT-3'. PCR and cloning sequencing conditions were standardized as described above. RT-PCR product was visualized and purified on a $2 \%$ agarose gels.

\section{Results}

Six $P T C H$ mutations in 3 NBCCS-associated and 2 sporadic KCOTs were identified, four of which were novel. The 6 mutations consisted of 2 nonsense, 3 frameshift and 1 inframe duplication mutations. A summary of the results obtained is shown in Table 1.

\section{Mutations in sporadic KCOTs}

We identified 2 somatic mutations in 8 sporadic KCOTs. One known frameshift mutation (c.3162 dupG) in exon 18 was detected in $\mathrm{KC} 1$ (Figure 1), which was predicted to introduce a stop codon at amino acid residue 1144, located at the sixth extracellular loop of PTCH protein. One novel inframeshift mutation was identified (c.1362-1374 dup) in exon 10 of patient $\mathrm{KC} 2$. Cloning sequencing revealed a duplication of $12 \mathrm{bp}$ nucleotides. It led to a four amino acid duplication in sterolsensing domain of PTCH protein and was highly conserved across species through alignment of the amino acid sequences of protein orthologs of human (Hs), chicken $(\mathrm{Gg})$, mouse $(\mathrm{Mm})$, Xenopus (Xl), and zebra fish (Dr) (GenBank Accession No: U59464.1, U40074, U46155, AF302765, and AJ007742). 
Table 1 Summary of PTCH mutations in 8 sporadic and 4 NBCCS-associated KCOTs

\begin{tabular}{|c|c|c|c|c|c|c|}
\hline Patient & Age/sex & Exon & Mutation $^{\mathrm{a}}$ & Effect on coding ${ }^{a}$ & Characteration & Patient's phenotype and history \\
\hline KC1 & $46 / M$ & 18 & c.3162dupG & p.D1055lfsX90 & Somatic, known & Multiple KCOTs, recurrent history \\
\hline $\mathrm{KC2}$ & $16 / M$ & 10 & c.1362-1374dup & p.L455-L458dup & Somatic, novel & Single KСОT \\
\hline NB1 & $53 / \mathrm{M}$ & 7 & c. $1012 \mathrm{C}>\mathrm{T}$ & p.Q338X & Somatic, novel & $\begin{array}{l}\text { Single KCOT, basal cell carcinomas of } \\
\text { skin, calcification of falx cerebri, bifid } \\
\text { rib, recurrent history }\end{array}$ \\
\hline NB2 & $15 / F$ & 14 & c.2179delT & p.C727VfsX19 & Germline, novel & $\begin{array}{l}\text { Multiple KCOTs, bifid rib, multiple skin } \\
\text { naevi, recurrent history }\end{array}$ \\
\hline NB3 & $26 / F$ & $\begin{array}{c}3 \\
17\end{array}$ & $\begin{array}{l}\text { c. } .403 \mathrm{C}>\mathrm{T} \\
\text { c.2824delC }\end{array}$ & $\begin{array}{l}\text { p.R135X } \\
\text { p.R942GfsX20 }\end{array}$ & $\begin{array}{l}\text { Somatic, known } \\
\text { Germline, novel }\end{array}$ & $\begin{array}{l}\text { Multiple KCOTs, calcification of falx } \\
\text { cerebri, bridged sella, frontal bossing, } \\
\text { palmar/planter pits, multiple skin naevi, } \\
\text { recurrent history }\end{array}$ \\
\hline
\end{tabular}

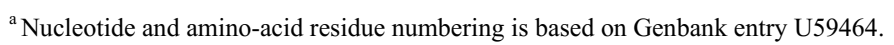

\section{Mutations in NBCCS-associated KCOTs}

A total of 4 mutations ( 3 novel and 1 known) were detected in 3 patients with NBCCS. DNA sequencing from KCOT tissues and peripheral blood revealed two were germline mutations and two were somatic mutations. Patient NB1, a 53-year-old male patient with typical NBCCS phenotypes, carried a nonsense mutation in exon 7 (c.1012C $>\mathrm{T}$ ). The mutation introduces a stop codon and leads to a premature termination at codon 338 . This mutation was somatic due to the absence from peripheral blood. Cloning sequencing of samples

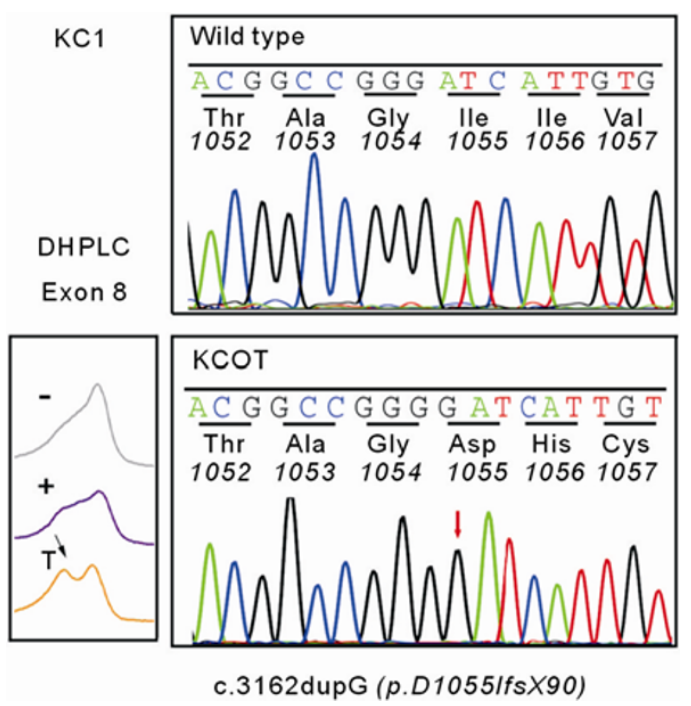

Figure $1 P T C H$ mutations in $\mathrm{KC1}$

A frameshift mutation (c.3162dupG, arrow) was detected in patient $\mathrm{KC} 1$, which introducing a stop codon at amino acid residue 1144 from NB2 revealed a threonine deletion at nucleotide 2179 in exon 14. This frameshift mutation introduces a stop codon at amino acid residue 745 , located in the third intracellular loop. Two mutations were identified in patient NB3, one is a germline frameshift mutation in exon 17 (c.2824 delC) and the other is a somatic nonsense mutation in exon 3 (c. $403 \mathrm{C}>\mathrm{T}$ ), resulting in premature stops at codon 961 and 135 respectively (Figure 2A). RT-PCR and cloning sequencing revealed these two mutations occurred in the same allele of the $\mathrm{PTCH}$ gene (Figure 2B).

\section{Discussion}

PTCH, which has been described as a tumor suppressor gene, is predicted to participate in tumorigenesis in NBCCS and related sporadic neoplasms. So far, several studies have provided evidence that $P T C H$ might take part in the pathogenesis of NBCCS-associated and sporadic KCOTs (Barreto et al., 2000; Gu et al., 2006; Sun et al., 2008). In this study, we identified 6 additional $P T C H$ mutations in 2 out of 8 sporadic and 3 out of 4 NBCCS-associated KCOTs, consisted of 2 nonsense, 3 frameshift and 1 inframe duplication mutations. The 2 somatic nonsense mutations, happened in exon 3 and 7 (c. $403 \mathrm{C}>\mathrm{T}$ and $\mathrm{c} .1012 \mathrm{C}>\mathrm{T}$ ), both resulted in $\mathrm{PTCH}$ protein premature stop at the first large extracellular loop, which has been summarized as one of the "hot" spots associated with hedgehog ligand binding 
Pan et al. Identification of PTCH Mutations in Keratocystic Odontogenic Tumors

(Lindstrom et al. 2006). Of the 3 frameshift mutations, 2 novel germline mutations in patient NB2 and NB3 (c.2179delT and c.2824delC) were predicted to lead to premature termination of PTCH at the large intracellular loop and the second large extracellular loop respectively. Patient $\mathrm{KC1}$ had multiple KCOTs but did not have other feathers of NBCCS. A somatic frameshift mutation (c.3162 dupG) in $\mathrm{KC1}$ was predicted to introduce a stop codon at amino acid residue 1144 , located at the sixth extracellular loop of PTCH protein. The inframe duplication mutation (c.1362-1374dup) in a 16-year-old patient (KC2) with a single KCOT caused a four amino acid duplication at the junction of transmembrane 2 and intracellular loop 1 of the PTCH protein. Transmembrane domains 2-6 were described as sterol-sensing domain and highly conserved across species. It is also a "hot" region analyzed by $\mathrm{PTCH}$ mutation database (Lindstrom et al., 2006). Of the six mutations identified, five
(c.403C > T, c.1012C > T, c.2179delT, c.2824delC, c.3162dupG) resulted in truncation of the PTCH protein. These results were in consistent with the previous reports that most $\mathrm{PTCH}$ mutations lead to a premature termination of the protein ( $\mathrm{Li}$ et al., 2008; Wicking et al., 1997) .

It is interesting that a NBCCS patient (NB1) only harbored a somatic mutation (c.1012C $>\mathrm{T}$ ) in the KCOT sample while the mutation was absent from corresponding peripheral blood. Instead of postulating that there would be another germline mutation which failed to be detected due to methodology, we could speculate that this somatic mutation (p.Q338X) might occur early in embryonic development and represent germline mosaicism in consideration of his typical clinical manifestations, such as basal cell carcinomas, calcification of falx cerebri, bifid rib, and recurrent history.

The molecular analysis of NBCCS associated tumors showed that a "two-hit" hypothesis is appli-
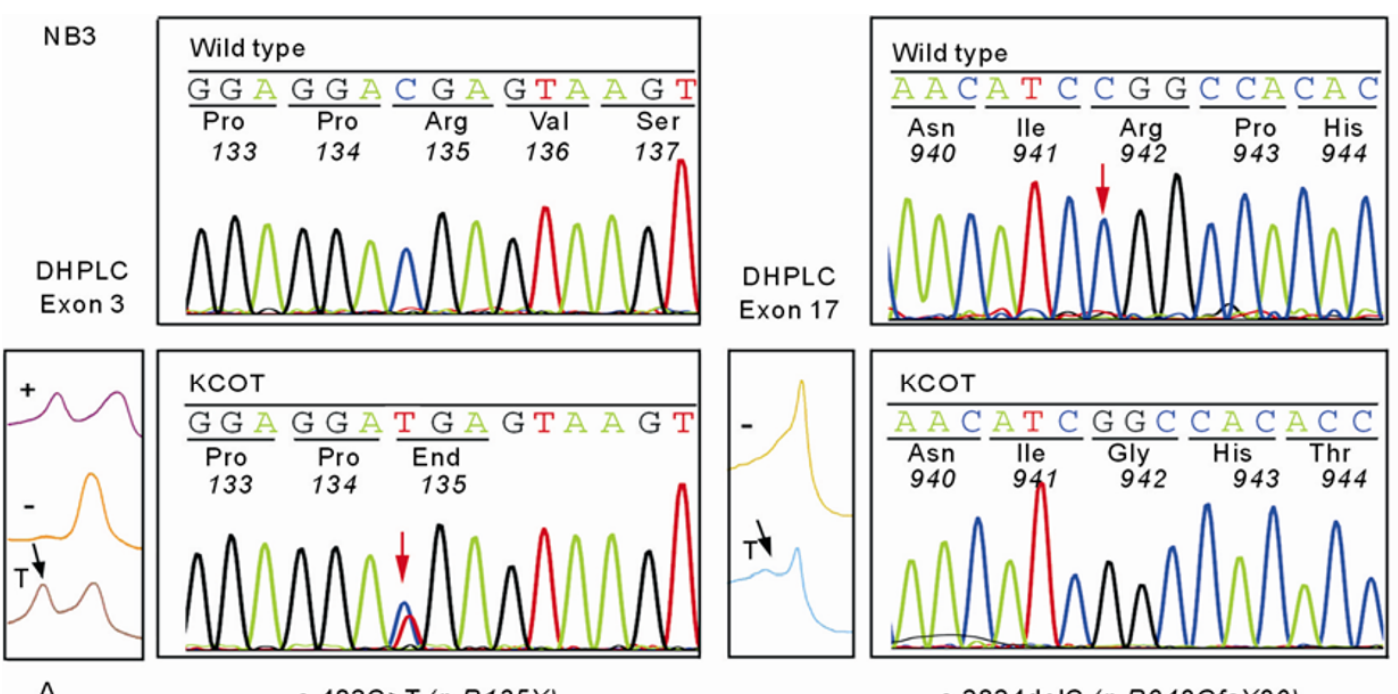

A

c. $403 C>T(p . R 135 X)$

c.2824delC (p.R942Gfs X20)
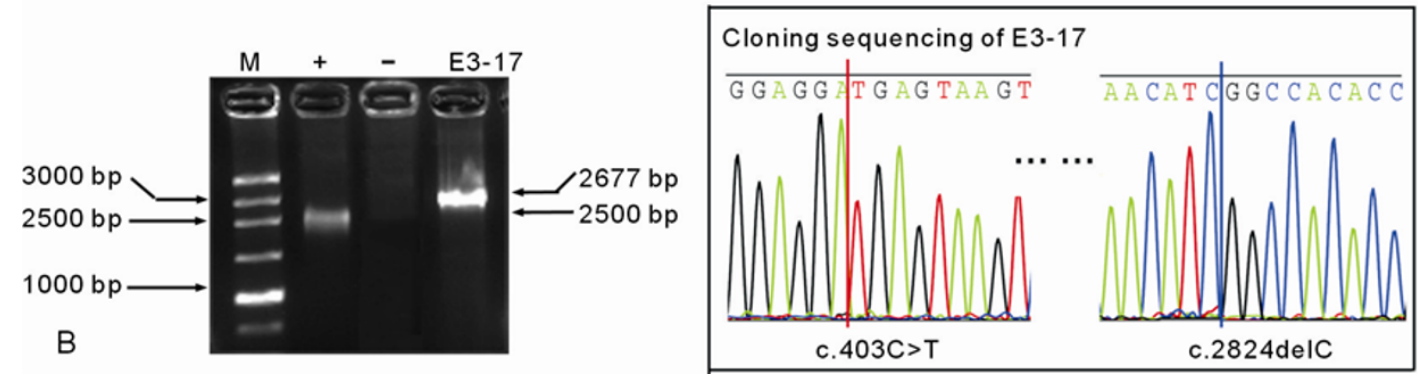

Figure $2 P T C H$ mutations in NB3

(A): Two coincident mutations were identified in NB3. One is a somatic nonsense mutation in exon 3 (c.403C $>$ T, arrow) which caused a stop at codon 135, the other is a germline frameshift mutation (c.2824delC) in exon 17. (B): RT-PCR amplified fragment (2677bp) spanning the region from exon 3 to exon 17 (left) and cloning sequencing of E3-17 indicated these two variants occurring in the same allele of $P T C H$ (right). 
cable to their pathogenesis (Levanat et al., 1996; Shear, 2002). These syndrome associated tumors presumably arise from precursor cells that contain a hereditary "first hit". Additional somatic mutation, loss of heterozygosity, or epigenetic silence of the other allele, acting as the "second hit", would introduce functional inactivity of $\mathrm{PTCH}$ protein. In addition to this, haploinsufficiency of PTCH is documented in several kinds of tumors, revealing that inactivation only one allele of PTCH gene might result in tumor genesis (Soufir et al., 2006). In the present study, we demonstrated a patient with syndrome (NB3) carried two different mutations (c.403C $>\mathrm{T}$, somatic and c.2824delC, germline). Further analysis by RT-PCR revealed that these two variants located in the same allele of $P T C H$. This result indicates the possibility that inactivity of $P T C H$ via haploinsufficiency and two-hit mechanism might both be involved in the pathogenesis of KCOTs.

\section{Conclusion}

In conclusion, $P T C H$ mutations could be identified in both sporadic and NBCCS-associated KCOTs. The result provides evidence for its crucial role in tumorigenesis of KCOTs even when they are not syndrome-related. Pathogenesis of KCOTs which did not carry PTCH mutations remains to be elucidated.

\section{Acknowledgements}

This work was supported by Research Grants from the National Nature Science Foundation of China (30625044, 30572048 and 30872900) and Specialized Research Fund for the Doctoral Program of Higher Education (20050001110). The authors also gratefully acknowledge the patients for their cooperation.

\section{References}

Bale AE, Yu KP (2001). The hedgehog pathway and basal cell carcinomas. Hum Mol Genet, 10(7): 757-762.

Barreto DC, Bale AE, De Marco L, Gomez RS (2000). PTCH gene mutations in odontogenic keratocysts. $J$ Dent Res, 79(6): 1418-1422.

Calzada-Wack J, Schnitzbauer U, Walch A, Wurster KH, Kappler R, Nathrath M, et al. (2002). Analysis of the PTCH coding region in human rhabdomyosarcoma. Hum Mut, 20(3): 233-234.

Gorlin RJ (2004). Nevoid basal cell carcinoma (Gorlin) syndrome. Genet in Med, 6 (6): 530-539.

Gu XM, Zhao HS, Sun LS, Li TJ(2006). PTCH mutations in sporadic and Gorlin-syndrome-related odontogenic keratocysts. J Dent Res, 85(9): 859-863.

Kimonis VE, Goldstein AM, Pastakia B, Yang ML, Kase R, DiGiovanna JJ, et al. (1997). Clinical manifestations in 105 patients with naveiod basal cell carcinoma sydrome. Am J Med Genet, 69(3): 299-308.

Levanat S, Gorlin RJ, Fallet S, Johnson DR, Fantasia JE, Bale AE (1996). A two-hit model for developmental defects in Gorlin syndrome. Nat Genet, 12 (1): 85-87.

Li TJ, Yuan JW, Gu XM, Sun LS, Zhao HS (2008). PTCH germline mutations in Chinese nevoid basal cell carcinoma syndrome patients. Oral Dis, 14(2): 174-179.

Lindström E, Shimokawa T, Toftgård R, Zaphiropoulos PG (2006). PTCH mutations: distribution and analyses. Hum Mut, 27(3): 215-219.

Pietsch T, Waha A, Koch A, Kraus J, Albrecht S, Tonn J, et al. (1997). Medulloblastomas of the desmoplastic variant carry mutations of the human homologue of Drosophila patched. Cancer Res, 57 (11): 2085-2088.

Shear M (2002). The aggressive nature of the odontogenic keratocyst: is it a benign cystic neoplasm? Part 2 . Proliferation and genetic studies. Oral Oncol, 38(4): 323-331.

Soufir N, Gerard B, Portela M, Brice A, Liboutet M, Saiag $\mathrm{P}$, et al. (2006). PTCH mutations and deletions in patients with typical nevoid basal cell carcinoma syndrome and in patients with a suspected genetic predisposition to basal cell carcinoma: A French study. Brit J Cancer, 95(4): 548-553.

*Corresponding author: Tie-jun Li

Address: Department of Oral Pathology, Peking University, School and Hospital of Stomatology, 22 South Zhongguancun

Avenue, Beijing 100081, China

Tel: 861062179977 ext $2203 \quad$ Fax: $861062173402 \quad$ E-mail: litiejun22@vip.sina.com 